\title{
Campus Displacement Experiences of the University of the Philippines Students and Teachers Affected by Typhoon Haiyan: Perceived Needs for Mental Health and Psychosocial Support
}

\author{
Anna Cristina A. Tuazon \\ University of the Philippines Diliman
}

\begin{abstract}
Background. Typhoon Haiyan made landfall over the Visayas Region in 2013, affecting 15 million people. At least 4 million people were displaced, including hundreds of University of the Philippines students and teachers who had to deal with the consequences of such displacement not only on their personal lives but also on their academic lives.

Objective. This study explored the experiences and needs of students and teachers of the University of the Philippines Visayas Tacloban College (UP VTC), University of the Philippines Manila School of Health Sciences (UPM SHS), and University of the Philippines Diliman (UPD) who were either directly affected by Typhoon Haiyan or served as responders to the typhoon survivors. The study specifically looked at experiences of displacement in an academic setting, from the perspective of those who were academically displaced and those who hosted them.
\end{abstract}

Methods. A qualitative descriptive study was designed involving a total of 17 student and teacher survivors and responders (ten survivors or survivor-responders and seven responders), who were purposively sampled and participated in an online open-ended questionnaire that elicited narrative experiences post-Typhoon Haiyan. Archived group process notes during the Haiyan response were also included as data. Qualitative thematic analysis was used to identify salient themes among and within groups.

Results. Salient themes of student and teacher survivors, survivor-responders, (i.e. survivors who also had the additional role of being responders for others), and responders that emerged included: (1) provision of basic needs (food, water, safety) including academic resources, (2) lack of deliberate psychosocial processing of the disaster experience for both survivor and responder, (3) unequal access to help, (4) communication and organizational problems, and (5) victimhood.

Conclusion. Student and teacher survivors and survivor-responders cited needs that go beyond basic survival needs that require a more contextual approach. Given the university context of student and teacher survivors, survivorresponders, and responders, recommendations included the need for (1) efficient dissemination of existing postdisaster school policies and programs to increase access to address communication and organizational issues, (2) equal access to school-based basic, financial, educational, and psychological support and services, and (3) proper sensitivity training for host students, teachers, and staff to decrease experiences of victimhood and discrimination.

Key Words: disaster experience, disaster victims, mental health, Philippines, psychosocial support

Corresponding author: Anna Cristina A. Tuazon, PsyD

Department of Psychology, College of Social Sciences and Philosophy,

University of the Philippines Diliman, Quezon City, Philippines

Telephone: +6329282728

Email: aatuazon@up.edu.ph 


\section{INTRODUCTION}

On November 2013, Typhoon Haiyan (Philippine name: Yolanda) made landfall over the Visayas Region with a recorded wind speed of more than $300 \mathrm{kph}$, making it the fourth strongest cyclone in recorded history. ${ }^{1,2}$ Almost 15 million people were affected by Typhoon Haiyan, with over 4 million displaced from their homes. ${ }^{3}$ Several of the schools directly affected by Typhoon Haiyan included the University of the Philippines Visayas Tacloban College (UP VTC) and the University of the Philippines Manila School of Health Sciences (UPM SHS) in Leyte, both of which are part of the University of the Philippines (UP) System. ${ }^{4}$ UP is the national university of the Philippines, with campuses across the country. Hundreds of students from UP VTC opted to temporarily transfer to the Diliman campus in Metro Manila while waiting for UP VTC to reopen. Students from UPM SHS, whose campus was destroyed, were housed in UP VTC after its reopening. ${ }^{4}$

Much of the focus on mental health and psychosocial support (MHPSS) services research were on short-term interventions like psychological first aid (PFA) and critical incident stress-debriefing (CISD). ${ }^{5}$ Psychological first aid is administered to survivors, typically within two weeks of a disaster, with focus on establishing safety and security, identifying coping strategies, and linking survivors to other needed services. ${ }^{6,7}$ Critical incident stress debriefing, on the other hand, is designed for responders and focused mainly on processing thoughts and feelings regarding the disaster event. ${ }^{8}$ CISD had been widely criticized for potentially increasing symptoms of posttraumatic stress and its misapplication to survivors ${ }^{8}$ while PFA had been increasingly adopted by international agencies such as the World Health Organization and the Red Cross. ${ }^{9,10,11}$ These interventions, however, only addressed the immediate aftermath of a disaster. Whereas the Inter-Agency Standing Committee (IASC) recommended that any comprehensive MHPSS system should cover a minimum of three years post-disaster ${ }^{12}$, most MHPSS delivery cannot be sustained for this long. This may be partly due to MHPSS delivery being attached primarily to the work and responsibility of emergency and disaster responders who, by nature of their work, must move on to other disasters and emergencies.

One potential under-tapped resource, however, for midto long-term MHPSS interventions for student survivors is the college or university campus. College campuses already provide year-round housing to their students, faculty, and staff, and provide routine access to basic needs such as food, health, scholarships, and counselling services. Moreover, the University of the Philippines has committed to the implementation of the disaster risk and reduction management (DRRM) framework through the creation of DRRM committees, subcommittees, and unit committees mandated to develop and implement DRRM policies. ${ }^{13}$ This indicates that the university considers disaster risk and reduction management as one of its responsibilities. As MHPSS is an integral component of any DRRM framework, this must be addressed as well. There is also no scarcity of human resources. A significant number of volunteer activities and outreaches routinely occur in an academic environment, largely due to student organizations and councils, as well as service learning requirements in the classrooms. College campuses also have on their rolls a wide variety of experts in any given field, particularly those who have the capacity to significantly contribute to disaster response. Moreover, college campuses have an existing organizational structure to disseminate information and delegate tasks, making it feasible to incorporate disaster risk and reduction management.

However, there are complexities to providing mid- to long-term MHPSS to the academic population. Survivors not only include students but also faculty and staff. Student needs, for example, are not limited to basic physical needs but necessarily include developmental needs (e.g., need for independence) and academic support. ${ }^{12}$ Some students may still be underage, thus complicating matters of informed consent for services. ${ }^{9}$ Handling post-traumatic symptoms in the classroom may also require some consideration, such as provision of academic accommodations and teacher training on classroom-based MHPSS. ${ }^{14}$

In the context of disasters, displacement is defined as a necessary move to a safer location. Campus displacement, then, is defined as moving to another campus to resume academic functioning. ${ }^{9,15}$ As young adults, campus displacement also coincides with shifting peer socialization practices and the influence on their identity development. ${ }^{16,17}$

Given the resources and complexities of an academic environment and its population, it is important to study the specific experiences and needs of displaced students as well as the experiences of the MHPSS providers within academic institutions to develop appropriate and effective delivery systems of services. This study specifically explored the experiences and needs of University of the Philippines students and teachers (1) who were directly affected by Typhoon Haiyan, (2) who were displaced to another campus, and (3) who provided MHPSS within the university.

\section{METHODS}

\section{Participants}

As a qualitative descriptive study, narrative experiences were elicited online 3 months after the disaster from a total of 17 students and teachers belonging to the University of the Philippines System. Purposive sampling was employed, with emphasis on reaching out to students and faculty from UPVTC and UPM SHS, which were the campuses that had to relocate. Host students and faculty were represented by those from the UP Diliman and UPVTC campuses, which hosted UPVTC and UPM SHS students, respectively. Screening criteria included being 18 years or older and being 
a current student or teacher in the UP System. Specifically, there were 11 undergraduate students, two teachers, and four student-teachers, with a mean age of 28.19. Of these, 10 were directly affected by Typhoon Haiyan and seven of them were displaced to another campus. Three teachers stayed in their campus to act as MHPSS providers while the remaining seven acted solely as MHPSS providers in behalf of the host campus.

Group process notes of providers that were archived by the MHPSS program of the UP Department of Psychology's Wellness Committee were also mined for aggregate data (no clients were identified) and included in the overall analysis.

\section{Procedures}

An online open-ended questionnaire was formulated to elicit narrative experiences of students and teachers post-Typhoon Haiyan. Data were collected online to increase access for participants from provincial campuses and to further ensure anonymity and confidentiality of the participants. Explicit emphasis was placed on psychosocial needs and services received immediately after the disaster and during displacement. Questions were: (1) What were your immediate needs post-Typhoon Haiyan/Yolanda, (2) What were your needs and experiences as students or faculty post-Typhoon Haiyan/Yolanda, (3) How did you experience the UP community's response to disasters, if any, (4) What issues or challenges arose with regard to the UP community's response to Typhoon Haiyan/Yolanda, (5) What types of psychosocial services would be helpful postTyphoon Haiyan/Yolanda, (6) Were you actively involved in the UP community's response to disasters and describe your experience, (7) What issues or challenges arose with regard to being a respondent/provider for the UP community, (8) If you cross-registered or transferred to another campus, please describe your experience with the transition and stay at the host campus, and (9) Do you have any recommendations as to how UP can respond better to its constituents' needs in times of disaster and describe.

Informed consent was obtained online, with a feature that would not allow them to proceed to the questionnaire if they did not specifically check the box giving consent. The informed consent provided information on the objectives of the study, the nature of the questions to be asked, guarantee of anonymity, and safeguards in case any of the questions will trigger a reaction; a clinical psychology team was on standby, and contact information were provided in the consent form. The nature of the questions themselves was purposefully general to prevent any re-enactments of participants' trauma reactions. The study proposal underwent an ethics review and was approved by the Clinical Psychology program of the UP Diliman Department of Psychology before implementation.

Demographic questions were also asked, including whether they were a student or faculty, the campus they were affiliated with, the campus they cross-registered in, age, gender, and area of study.
The archival data from the Department of Psychology's Wellness Committee were obtained with permission from the Chair of the committee. As these were process and not progress notes, the psychology code of ethics (Psychological Association of the Philippines, 2002) conferred rights to such notes to the clinicians and to the committee. ${ }^{18}$

\section{Analysis}

Qualitative thematic analysis was used to identify salient themes among and within groups; involved were three encoders to reach consensus. The researcher and two other Psychology graduates who were trained in qualitative research comprised the encoders. Using the written responses in the questionnaire, all key words were identified and coded. These key words were then grouped with other related key words until the initial themes were formed. These initial themes were then grouped into master themes.

The archival data comprised of four group process notes, which also underwent the same thematic analysis as above. All key words were identified and coded, organized into initial themes, and further organized into master themes. These were then compared to the initial and master themes conducted for the online responses and were aligned. Themes from the archival data that were not identified by the online respondents were added instead of removed, in keeping with the objective of qualitative analysis of obtaining a wide range of responses and themes.

\section{RESULTS AND DISCUSSION}

Qualitative thematic analysis yielded five master themes: (1) basic needs, (2) psychosocial, (3) unequal access to help, (4) communication, coordination and organizational problems, and (5) victimhood.

\section{Basic Needs}

Basic needs were further divided into eight sub-themes: (1) food and water ( $n=10),(2)$ medicine ( $n=9)$, (3) housing and utilities $(n=7),(4)$ security $(n=2),(5)$ clothing $(n=2),(6)$ financial $(n=5),(7)$ transportation $(n=3)$, and (8) academic $(n=14)$.

Basic needs of students and faculty directly affected by Typhoon Haiyan were the most commonly cited. Notably, they also identified academic resources as part of their basic needs. UPVTC students noted that their "classrooms were not that comfortable and conducive to learning", that they were "staying in very hot tents", and that they "badly needed internet connection" for their academic requirements. Both online responses and archived process notes also mentioned having to adjust to a bigger campus, which required having to be acquainted with more students, new teachers, and different pedagogies. While current DRRM strategies outside of universities are capable of addressing basic survival needs, host universities are uniquely positioned to address academic concerns which are top of mind for student 
survivors. Aside from material and logistical needs related to academic learning, proper academic orientation to the host campus to ease transition and minimize adjustment issues were also identified by student survivors.

\section{Psychosocial Needs}

Psychosocial needs were cited by 15 out of 17 respondents. Both student and teacher survivors cited the need for deliberate processing of their disaster experience. Some UPVTC students and teachers reported the need for "sustainable counseling or therapy service" while others indicated that "group psychotherapy would be most helpful". Other students stated a need for a "safe space to share our feelings, not only about our experience in Tacloban but in the aftermath, as we go and try to function and cope." Universities' counseling centers can provide avenues for emergency counseling for survivors and spare rooms can be temporarily converted to safe spaces, similar to spaces reserved for student lounges.

It is important to note that some student and teacher survivors stated not having received any form of processing and were instead expected to act as disaster responders. One UPM SHS student stated that "we were involved in postdisaster work despite the fact that we were survivors ourselves and we were only processed more than a month after the typhoon." A UPVTC teacher responded that "psychosocial assistance was lacking... we only got to be processed by another university." This resulted in prolonged experiences of stress, frustration, and anger, with some teacher survivors stating experiencing "burnout". They further reported that "We were responding to so many things practically every day despite the fact that we were survivors ourselves," while another respondent wrote "I went through all the motions of listening but I knew deep down that I also needed to unload". Another teacher survivor narrated re-experiencing trauma and stated that "sometimes listening to the sharing of the students, I would relive the experience again and again." The participants' stated need for deliberate processing and the consequences of the lack thereof is supported by Devilly et al (2006) who looked at the impact of psychological debriefing and recommended that instrumental support and assistance be offered immediately after a disaster. ${ }^{8}$ Based on these experiences of survivor-responders, it is important to include debriefing or processing for both survivors and responders as part of any DRRM protocol.

For participants who served as responders, students cited the need for more formal training on being a psychosocial support provider while teachers identified the need to balance multiple roles and responsibilities within the university including that of being a responder. Teacher responders cited experiencing burnout after doing MHPSS work. One teacher noted, "Firstly, there were limited resources that affected capacity to establish comprehensive network of support sources, provide more comprehensive psychological services relevant to student life, and track students closely and making services accessible... this was frankly quite frustrating." Burnout among responders is common, with potential for compassion fatigue and secondary traumatic stress. ${ }^{21,22}$ More specifically, Lewis and Gills (2008) saw the importance of preparedness and the development of a disaster network at school in minimizing trauma symptoms. ${ }^{23}$ This significant protective factor against burnout was already mentioned by student responders - training. This should be feasible for universities. For example, the University of the Philippines Diliman already offers a course on DRRM and on disaster mental health interventions. Further development of DRRM strategies and systematizing policies, procedures, and protocols can help in teacher preparedness.

Many participants also emphasized needing to receive emotional support from their university, citing how that could increase their sense of hopefulness and control over the post-disaster situation. One UPM SHS student survivorresponder wrote, "I guess what we just wanted was to hear and be assured that everything will be alright and the UP System is there for us." This is in keeping with a sense of community playing a protective role on emergency workers' quality of life. ${ }^{18}$ This means that more than provision of logistical resources, universities can work on inspiring a sense of hope and control to their constituents. This can be done, at a minimum, with mere presence of university officials to signify concern and involvement. This can also be further enforced by direct and public messages of comfort.

\section{Unequal Access to Help}

Another emerging theme specific to an academic environment was a perception of unequal access to support and services, which was cited by seven participants. A typical response in this category was "bindi same yung support ng different campus sa victims/survivors ng Yolanda (Support was not the same across campuses for victims/survivors of Yolanda)." Moreover, several student survivor participants cited experiences of inter-campus tensions, preferential treatment, and experiences of direct discrimination from hosts with one student sharing an experience where a host student pointed out her clothes and asked if it was a donation. Universities can address these inequalities by streamlining DRRM policies and interventions across campuses. Discrimination can be minimized through proper and explicit orientation of host campuses including teachers and students on how to ensure inclusivity and reinforcing the sense of community for the entire system, not just per campus.

\section{Communication, Coordination, and Organizational Problems}

Communication, coordination, and organizational problems were cited by 13 participants. A teacher responder noted that "information was not easily or effectively disseminated because the campus units are traditionally used to working as islands, making for quite a siloed organization, 
and information falling through communication cracks at a time when timely communication was paramount." Student survivors noted that a "delay in getting information also at times deprived some students of certain assistance." Such problems are common, with Osofsky (2008) recounting similar experiences during Hurricane Katrina. ${ }^{20}$ This can be addressed by implementing, through the DRRM framework, a university system-wide chain of command (which should also necessitate proper DRRM training for administrative officials who will serve as command) to ensure proper and systematic dissemination of any needed information regarding assistance and services.

\section{Victimhood}

Finally, themes of victimhood were echoed by seven student survivor participants, stating their wish not to be identified as victims or even as survivors but simply as students. Some responses pertained to focusing on a role other than victim to combat feelings of helplessness, such as helping with relief operations and medical missions. Identity development is of particular concern among college-aged students and disasters can affect their sense of identity and autonomy. ${ }^{15}{ }^{24}$ Moreover, excessive focus on deficits and victimhood may impede resilience among student survivors. ${ }^{25}$ This can be addressed by the university by providing avenues and opportunities for people to sign up or volunteer for relief tasks or to help survivors get back on track with their academic coursework as soon as possible.

\section{CONCLUSION AND RECOMMENDATIONS}

Typhoon Haiyan brought about, among other devastating consequences, displacement of hundreds of students and teachers who had to be housed in other campuses to continue their academic lives. This presents a complex scenario where provision of basic needs is not enough; academic, developmental, and emotional needs must be addressed as well.

The identified themes highlight needs and concerns that can only be addressed in an academic setting such as the importance of upholding survivors' identity as student, regaining a sense of normalcy and control by resuming academic activities and responsibilities, and the need for emotional assurance specifically from their academic community.

Such results pose a challenge to academic institutions who wish to respond effectively to their students and teachers in times of disaster: how can all these needs be addressed in a manner that does not emphasize victimhood, that minimizes discriminating and preferential attitudes by the host campus, and that prevents burnout by the MHPSS providers or responders? One way to address these challenges is through the systematic and consistent implementation of the DRRM framework, with emphasis on MHPSS. Proper implementation of DRRM necessitates good preparation: formal and regular training for all types of providers including psychosocial support providers and development of a response program that should include a centralized system of relief services and programs. This would minimize inequality of access to such services and lessen inter-campus tensions and conflicts. Proper implementation of DRRM and MHPSS should also always include psychosocial processing or debriefing to prevent trauma symptoms for survivors and to prevent burnout for responders.

As universities and other academic institutions are committing themselves to the DRRM framework, it is their duty to respond to the needs of students and teachers in times of disaster. Especially since the Philippines is expected to have multiple disasters in any given year, being prepared is essential. As an environment that physically houses students throughout their academic lives, universities are contextually well-placed to serve mid- to long-term needs of displaced student survivors as compared to any other venue. As it currently stands, universities already have pre-existing structures and services for students and teachers (e.g.housing, food, libraries, guidance and counseling centers, health centers), and have pre-existing organizational systems that can be used to delegate tasks and disseminate information. During times of disaster, these structures and services can be augmented via the larger university community such as the surrounding residents, alumni, and host students and their families that a university should have ready access to. Both literature and current findings support the need for students to resume student life as soon as possible to facilitate recovery, and the imperative to place them back into supportive academic settings.

Limitations of the study include the descriptive and qualitative nature of the study, so that results cannot be considered representative of all student and teacher concerns post-disaster. Also, since all the participants came from the same university system, their responses may unduly reflect a specific school or regional culture. While online data collection is considered to elicit more truthful and unbiased responses, this method of data collection does not allow for probing or elaboration of their narratives which means that certain themes that are otherwise salient were not articulated or identified. Recommendations for future research include using quantitative approaches to confirm if addressing the identified psychosocial needs would lead to increased resilience, less trauma symptoms, and less burnout or fatigue.

While provision of basic needs is part of any DRRM implementation, psychosocial needs should be prioritized as well. Aside from access and availability of direct psychosocial services, this also must include: (1) efficient dissemination of information that contain statements of support, (2) equal access to support and services with minimized bureaucratic barriers in times of disaster, and (3) proper sensitivity training for host students, teachers, and staff to decrease incidences of discrimination and to decrease the forced sense of victimhood. 


\section{Acknowledgments}

The author would like to thank the University of the Philippines Department of Psychology Wellness Committee for permitting the use of their archival records. The author would also like to thank Claire Aragon, Lizette Inocencio, Katherine Castro, Jerieka Fernandez, and Kim Salvador for their invaluable assistance to this study.

\section{Statement of Authorship}

The author has approved the final version submitted.

\section{Author Disclosure}

The author has declared no conflict of interest.

\section{Funding Source}

No external funding.

\section{REFERENCES}

1. Lagmay A, Agaton R, Bahala M, et al. Devastating storm surges of Typhoon Haiyan. International Journal of Disaster Risk Reduction. 2015; 11: 1-12.

2. https://doi.org/10.1016/j.ijdrr.2014.10.006

3. Sedghi A. Typhoon Haiyan: how does it compare with other tropical cyclones?. The Guardian. 2013 Nov 8.

4. National Disaster Risk Reduction and Management Council. Update on the effects of Typhoon Yolanda [Online]. 2014 [cited2015 September]. Available from: http://ndrrmc.gov.ph/attachments/ article/1329/Update_on_Effects_Typhoon_YOLANDA_ (Haiyan)_17APR2014.pdf.

5. Llaneta $\mathrm{C}$. The day the Oblation stood still: UP responds to the challenge of Typhoon Yolanda [Online]. 2014 May 7 [cited 2015 September]. Available from http://web-old.up.edu.ph/the-day-theoblation-stood-still-up-responds-to-the-challenge-of-typhoonyolanda-2/.

6. Meyer S. UNHCR's mental health and psychosocial support for people with concerns. Geneva: United Nations High Commissioner for Refugees, Policy Development \& Evaluation Service; 2013. pp. 55-72.

7. Brymer M, Layne C, Jacobs A, et al. Psychological first aid: field operations guide. 2nd ed. California, USA: National Child Traumatic Stress Network and National Center for PTSD; 2006.

8. Paya PM. Understanding the need for mental health and psychosocial support in times of emergency. Asian Disaster Management News. 2013;20(2013):6-7.

9. Devilly G, Gist R, Cotton P. Ready! Fire! Aim! The status of psychological debriefing and therapeutic interventions: in the work place and after disasters. Review of General Psychology. 2006;10(4):318-45. doi.org/10.1037/1089-2680.10.4.318.

10. World Health Organization, War Trauma Foundation and World Vision International. Psychological first aid: guide for field workers. WHO: Geneva; 2011.
11. International Federation of Red Cross and Red Crescent Societies, \& Reference Center for Psychosocial Support. Psychosocial interventions: a handbook. Copenhagen, Denmark: International Federation Reference Centre for Psychosocial Support; 2009.

12. Australian Red Cross \& Australian Psychological Society. Psychological first aid: an Australian guide to supporting people affected by disaster. Victoria, Australia: Australian Red Cross \& Australian Psychological Society; 2013.

13. Inter-Agency Standing Committee. IASC guidelines on mental health and psychosocial support in emergency settings. Geneva: IASC; 2007.

14. Sanvictores, BV. Building arts, linking cultures. Bridges: An UPDate Magazine Special Edition. 2016; 3(2): 20-9.

15. The Institute for Trauma and Stress. Caring for kids after trauma and death: a guide for parents and professionals. New York: The Institute for Trauma and Stress; 2002. Available from http://www.nctsn.org/ nctsn_assets/pdfs/Crisis\%20Guide\%20-\%20NYU.pdf

16. Tuason MTG, Guss CD, Carroll L. The disaster continues: a qualitative study on the experiences of displaced Hurricane Katrina survivors. Professional Psychology: Research and Practice. 2012; 43(4): 288-97.

17. Forbes RJ, Jones R, Reupert A. In the wake of the 2009 Gippsland fires: young adults' perceptions of post-disaster social supports. Aust J Rural Health. 2012; 20(3):119-25. doi: 10.1111/j.1440-1584.2012.01271.x.

18. Pena-Alampay L. Self-complexity, self-construal, and negative emotion in Filipino adolescents. Philippine Journal of Psychology. 2003; 36(2): 68-102.

19. Psychological Association of the Philippines. Code of ethics for Philippine psychologists. 2008 [cited 2017 May]. Available from: https://www.pap.org.ph/includes/view/default/uploads/code_of_ ethics_pdf.

20. Cicognani E, Pietrantoni L, Palestini L, Prati G. Emergency workers' quality of life: the protective role of sense of community, efficacy, beliefs and coping strategies. Social Indicators Research. 2009; 94: 499.

21. Osofsky JD. In the aftermath of Hurricane Katrina: a personal story of a psychologist from New Orleans. Professional Psychology: Research and Practice. 2008; 39(1): 12-7. http://dx.doi.org/10.1037/07357028.39.1.12

22. Alexander DA, Klein S. First responders after disasters: a review of stress reactions, at-risk vulnerability, and resilience factors. Prehospital and Disaster Medicine. 2009; 24(2): 87-94. https://doi.org/10.1017/ S1049023X00006610.

23. Naturale A. Secondary traumatic stress in social workers responding to disasters: reports from the field. Clinical Social Work Journal. 2007; 35(3): 173-81

24. Lewis JS, Gillis HJ. Reflections from the field: on the road to recovery. Traumatology. 2008; 14(4): 55-62. https://doi. org/10.1177/1534765608326179.

25. Boyer E. A student social worker's reflection of the self and professional identity following the impact of Hurricane Katrina on New Orleans. Traumatology. 2008; 14(4): 32-7. https://doi. org/10.1177/1534765608325296.

26. Wessells MG. Do no harm: toward contextually appropriate psychosocial support in international emergencies. Am Psychol. 2009; 64(8):842-54. doi: 10.1037/0003-066X.64.8.842. 